\title{
Hydration of the Zwitterionic Forms of Amino Acids from the Acoustic Passynski Method
}

\author{
A. Burakowski and J. Gliński* \\ Faculty of Chemistry, University of Wrocław \\ F. Joliot-Curie 14, 50-383 Wrocław, Poland
}

In this communication, we report the compressibility measurements made on amino acids in water at $25^{\circ} \mathrm{C}$, compared with aqueous short-chained amines and carboxylic acids. The effect of the charge caused by protonation of the amino group and/or deprotonation of the carboxylic one was tested. The concept of additivity of the hydration number with the constituents of solute molecule was applied and discussed.

PACS numbers: 43.35.Bf, 43.35.+d, 61.20.-p, 61.20.Qg

\section{Introduction}

The interest of the biophysical community toward acoustics, in particular compressibility as an independent and effective tool for investigating biologically active compounds have begun to find an increasing employment as means for characterizing protein systems $[1,2]$.

Most biological macromolecules are physiologically active in aqueous solutions. Thus knowledge of water-protein interaction is necessary to understand the role of water solvated to soluble organics in the living cells. Since amino acids exist in zwitterionic forms in aqueous solutions, their volume and compressibility properties should reflect structural interactions with water molecules as it is observed in the case of electrolytes.

Investigations of the volume properties of proteins and some amino acids have shown that there is a decrease in volume upon dissolving these compounds in water $[3,4]$. This decrease in volume, similar to what occurs for electrolytes, can be attributed to electrostriction due to water-solute interactions. The above might indicate that amino acids or proteins behave as electrolytes, however, it seems rather that the so-called "bound water" behaves more like water solvated to soluble organic solutes $[5,6]$.

*corresponding author; e-mail: glin@wchuwr.pl 


\section{Experimental}

Solutions were prepared by weighing before measurements using doubly distilled, fresh-prepared water. All the chemicals were of highest quality and used without additional purification. The compounds tested, the first members of homologic monocarboxylic acids, amines and amino acids are listed below in Table II.

Sound velocity was determined using a computer-steered OPKUD 01/100 (Optel, Poland) apparatus, with the absolute accuracy better than $\pm 0.2 \mathrm{~m} \mathrm{~s}^{-1}$ and the precision of similar order. Measurements are based on the determination of the time an acoustic signal needs to pass through the sample of known length.

Density was measured using a vibrating tube Ecolab MG-2 (Kraków, Poland) apparatus with an accuracy $c a . \pm 0.1 \mathrm{~kg} \mathrm{~m}^{-3}$.

Temperature of the measurements was $25^{\circ} \mathrm{C} \pm 0.05$, stabilized by a precision MLW UH (Germany) thermostat. Its stability was controlled by digital thermometer built in the density apparatus, and the absolute value by a precision mercury thermometer.

From the sound velocity and density data the adiabatic compressibility coefficients can be calculated, using the Laplace equation

$$
\kappa_{\mathrm{S}}=d^{-1} c^{-2}
$$

(where $d$ - density, $c$ - velocity of sound). The relative change of compressibility was assumed by Passynski as caused by engaging some fraction of water molecules in hydration spheres, where they become incompressible. This leads to the well-known formula for the hydration number, $n_{\mathrm{h}}$, known as the Passynski equation $[7,8]$ :

$$
n_{\mathrm{h}} \cong \frac{n_{1}}{n_{2}}\left(1-\frac{\kappa}{\kappa_{1}^{0}}\right),
$$

where $n_{1}$ and $n_{2}$ are the number of moles of water and solute in solution, respectively, $\kappa$ is compressibility of the solution and $\kappa_{1}^{0}$ is that of pure solvent.

There were three series of experiments, differing by solvent: it was water, $0.2 \mathrm{M} \mathrm{HCl}$ and $0.2 \mathrm{M} \mathrm{NaOH}$. The two latter were prepared using Fixanal $\otimes_{\text {chemicals. }}$

\section{Results and discussion}

Only very dilute systems were investigated in this work. The mole fraction of solutes never exceeded 0.01, i.e. there were at least 100 water molecules per one solute molecule. Therefore, one can assume - at least on geometrical basis that significant interactions between the solute molecules are negligible, the same concerns interactions between water molecules belonging to two different solvation clouds.

It is worth noting that - in the investigated range of concentrations every one of solutes gives perfectly linear $\left(R^{2}\right.$ coefficient was never lower than 0.999) dependence of compressibility $\kappa$ on concentration at least in the limits of the experimental error, which is rather low). 
The above means that the hydration numbers of non-electrolytes obtained by the Passynski formula are constant up to the limit of linearity of compressibility with composition. These values are now assumed by us to be a sum of contributions from individual constituents of the solute molecule, as it was proposed recently [9].

Applying the idea of additivity of hydration numbers with the geometry of solute molecules to few homologous series of compounds (alcohols, acids and amines) yields a number of linear equations with the number of unknowns equal to the number of constituents, easy to be solved. Similar ideas were presented already by Hedwig et al. [10], Cheng and Rossky [11] and others [12, 13]. For instance, Inzelt and Grof found that in polyvinyl alcohols the hydration number is 3.7-4 per one $\mathrm{OH}$ group [14], i.e. they assume that it is additive with the chain length. Let us note that the hydration numbers obtained by the Passynski acoustic method and already reported in the above cited literature are in very good agreement with those presented in this work.

The results of fitting are collected in Table I (the values of the constituents' hydration numbers in water, $0.2 \mathrm{M} \mathrm{HCl}$ and $0.2 \mathrm{M} \mathrm{NaOH}$ solvents) and in Table II (total hydration numbers, experimental and calculated). For comparison, the contributions calculated for much wider range of solutes (but not including amino acids) from [9] are included in the last row.

TABLE I

Hydration numbers of functional groups of molecules, i.e. their contributions to the observed ones, of non-electrolytes in water, obtained by fitting as described in the text.

\begin{tabular}{c|c|c|c|c|c|c|c}
\hline \hline Group & $-\mathrm{NH}_{2}$ & $-\mathrm{NH}_{3}^{+}$ & $-\mathrm{COOH}$ & $-\mathrm{COO}^{-}$ & $-\mathrm{CH}_{3}$ & $>\mathrm{CH}_{2}$ & $>\mathrm{CH}-$ \\
\hline in water & 1.19 & 2.20 & 1.11 & 2.20 & 0.65 & 0.94 & 1.48 \\
in $0.2 \mathrm{M} \mathrm{HCl}$ & - & 2.36 & 1.25 & - & 0.64 & 0.94 & 0.96 \\
in $0.2 \mathrm{M} \mathrm{NaOH}$ & 0.49 & - & - & -1.70 & 1.36 & 1.05 & 1.18 \\
in water, data from [9] & 1.15 & - & 1.15 & - & 0.59 & 1.00 & 1.32 \\
\hline
\end{tabular}

${ }^{*}$ Amino acids were not included in this fitting.

Although the contributions of groups to the observed hydration number listed in Table I are somewhat different in different solvents, one should remember that they were calculated for rather limited number of systems and are only indicative. Moreover, the total hydration numbers determined by the Passynski method are sensitive to the experimental error. While our experiments were limited to very low solute concentrations and, consequently, the observed variability of density and/or sound speed was also limited, the experimental error could be sometimes relatively high.

Nevertheless, independent of the solvent (water, acid or base) the general trends are conserved. In particular, the contributions increase in the order 
TABLE II

Hydration numbers (experimental and calculated) of nonelectrolytes in water, $0.2 \mathrm{M} \mathrm{HCl}$ and $0.2 \mathrm{M} \mathrm{NaOH}$. The theoretical (calculated) hydration numbers, $n_{\mathrm{h}}$ (calc.), were obtained using values from Table I.

\begin{tabular}{c|c|c|c|c|r|r}
\hline \hline \multirow{2}{*}{ Solute } & \multicolumn{2}{|c|}{ water } & \multicolumn{2}{c|}{$0.2 \mathrm{M}$} & $\mathrm{HCl}$ & \multicolumn{2}{|c}{$0.2 \mathrm{M}$ NaOH } \\
\cline { 2 - 7 }$n_{\mathrm{h}}$ & exp. & calc. & exp. & calc. & exp. & calc. \\
\hline ammonia & 1.1 & 1.2 & 2.3 & 2.4 & 0.8 & 0.5 \\
methylamine & 1.7 & 1.8 & 3.3 & 3.0 & 1.7 & 1.8 \\
ethylamine & 3.2 & 2.8 & 3.6 & 3.9 & 3.0 & 2.9 \\
propylamine & 3.7 & 3.7 & 4.7 & 4.9 & 3.7 & 4.0 \\
butylamine & 4.6 & 4.7 & 5.9 & 5.8 & 4.7 & 5.0 \\
pentylamine & 5.5 & 5.6 & 7.0 & 6.8 & 5.5 & 6.1 \\
formic acid & 1.2 & 1.1 & 1.4 & 1.3 & -3.7 & -1.7 \\
acetic acid & 1.8 & 1.8 & 1.6 & 1.9 & -0.6 & -0.3 \\
propanoic acid & 2.7 & 2.7 & 2.9 & 2.8 & 1.3 & 0.7 \\
butanoic acid & 3.5 & 3.6 & 3.9 & 3.8 & 2.6 & 1.8 \\
glycine & 5.5 & 5.3 & 4.9 & 4.6 & 0.2 & -0.2 \\
L-alanine & 6.2 & 6.5 & 5.3 & 5.2 & 1.6 & 1.3 \\
L-valine & 8.5 & 8.7 & 6.7 & 6.8 & 3.9 & 3.9 \\
L-leucine & 9.7 & 9.6 & 7.9 & 7.8 & 4.7 & 4.9 \\
L-isoleucine & 9.8 & 9.6 & 7.7 & 7.8 & 5.0 & 4.9 \\
4-aminobutanoic acid & 7.2 & 7.2 & 6.0 & 6.4 & 2.3 & 1.9
\end{tabular}

$-\mathrm{CH}_{3}<,=\mathrm{CH}_{2}<, \equiv \mathrm{CH}$. The exception for $\mathrm{NaOH}$ solution needs confirmation from additional tests - this concerns particularly the shortest monocarboxylic acids (formic and acetic) in $0.2 \mathrm{M} \mathrm{NaOH}$. The homologous acids with longer hydrocarbon tails are better described by the mentioned additivity of the hydration numbers.

The most important are, however, the results for protonated amino group $\left(-\mathrm{NH}_{3}^{+}\right)$and deprotonated carboxylic one $\left(-\mathrm{COO}^{-}\right)$. The former is relatively high (2.20 or more), the latter changes depending on environment in the degree making any explanation impossible now. This could be caused either by exceptional behavior of the shortest acids as mentioned above. The relatively high value for $-\mathrm{NH}_{3}^{+}$can be explained in terms of electrostriction of neighboring water molecules and formation of strong hydrogen bond during protonation, which could affect cooperatively water molecules more distanced from the hydrate.

However, similar phenomena should occur also for hydrated carboxylic group. It is possible that in $\mathrm{NaOH}$ solution, deprotonation suppresses the hydrogen bond engaging the dissociated proton, but this decrease in the group contribution to hydration by a factor of one should be recompensated by the electric 
charge appearing and subsequent electrostriction of solvent. The above suggests that hydration spheres of small anions should exist and their hydration should be positive. For instance, it was found from the Raman spectroscopy combined with X-ray and neutron diffraction that hydration numbers of sodium cation and formate anion are almost the same (4.6 and 4.4, respectively) [15]. All these makes the negative values in Table I difficult to explain.

Inspection of Table II shows that the reproduction of hydration numbers when applying the assumed additivity of them with solute molecule constituents and applying the contributions determined and shown in Table I is surprisingly good. In some cases the reproductibility is apparently worse, and these cases have to be specifically explained.

Discordances are significant for $\mathrm{NaOH}$ solutions of amino acids. Let us note that amino acids with longer hydrocarbon chains are better reproduced by the mentioned additivity than shorter ones. On the other hand, separation of polar groups (the case of 4-aminobutanoic acid), also aggravates the fitting.

The latter corresponds well with evidently poor fitting for simple carboxylic acids in $\mathrm{NaOH}$. Because of very low concentrations of solutes, no specific solutesolute interactions can be responsible for this. Moreover, neither the increased ionic strength nor formation of charged groups in a molecule is responsible for this behavior, because it was observed only in $\mathrm{NaOH}$ solutions, but never in $\mathrm{HCl}$ ones. Thus, an explanation could be replacing of $\mathrm{OH}^{-}$with $\mathrm{R}-\mathrm{COO}^{-}$anions in the system, the former having exceptionally high ionic hydration number [16], but additional tests are necessary for solving this question, for instance experiments for a series of carboxylic salts in water and in aqueous solutions with fixed $\mathrm{pH}$ 's. These results will be presented in forthcoming papers.

\section{Acknowledgments}

This project was partially supported by the Ministry of Science and Higher Education of the Polish State (grant no. 4032/B/H03/2007/33).

\section{References}

[1] K. Gekko, H. Noguchi, J. Phys. Chem. 83, 2706 (1979).

[2] A.P. Sarvazyan, Annu. Rev. Biophys. Biophys. Chem. 20, 321 (1991).

[3] E. Berlin, M.J. Pallansch, J. Phys. Chem. 72, 1887 (1968).

[4] J. Bernhardt, H. Pauly, J. Phys. Chem. 79, 584 (1975).

[5] F.J. Millero, A. Lo Surdo, Ch. Shin, J. Phys. Chem. 82, 784 (1978).

[6] F.J. Millero, G.K. Ward, P. Chetirkin, J. Biol. Chem. 251, 4001 (1976).

[7] A. Passynski, Acta Physicochimica U.R.S.S. 8, 385 (1938).

[8] A. Passynski, Acta Physicochimica U.R.S.S. 22, 137 (1947).

[9] J. Gliński, A. Burakowski, Eur. Phys. J. Special Topics 154, 275 (2008).

[10] G.R. Hedwig, J.D. Hastie, H. Hoiland, J. Solution Chem. 25, 615 (1996). 
[11] Y.-K. Cheng, P.J. Rossky, Nature 392, 696 (1998).

[12] A.B. Schmidt, Biophys. Chem. 51, 393 (1994).

[13] Q. Liu, J.W. Brady, J. Phys. Chem. B 101, 1317 (1997).

[14] G. Inzelt, P. Grof, Acta Chim. Acad. Sci., Hung. 88, 13 (1976).

[15] Y. Kameda, T. Mori, T. Nishiyama, T. Usuki, O. Uemura, Bull. Chem. Soc. Jpn. 69, 1495 (1996).

[16] D.S. Allam, W.H. Lee, J. Chem. Soc. A , 426 (1966). 